\title{
Development of STEM-Holography
}

Fehmi S. Yasin ${ }^{1}$, Tyler R. Harvey ${ }^{1}$, Jordan J. Chess ${ }^{1}$, Jordan S. Pierce ${ }^{1}$, Benjamin J. McMorran ${ }^{1}$ 1. Department of Physics, University of Oregon, Eugene

We have developed a new technique we call STEM-holography, inspired by J. M. Cowley [1], to measure the phase imparted on an electron after transmission through thin, low-atomic number specimens via phase contrast.

Low-atomic number materials play a crucial role in life sciences, medicine, and the carbon energy cycle. However, our ability to image these materials at the atomic length scale is limited because they do not scatter electrons at high-angles in the same way a crystalline or high atomic number material does. Additionally, these materials are easily damaged under electron beam illumination. To get around these issues, bold efforts have been made in the fields of electron holography [2] and ptychography [3, 4], leading to myriad techniques that can potentially achieve sub-nanometer resolution. Additionally, offaxis electron holography has been developed and applied in many research groups [5 - 8], pushing the boundaries of electron microscopy with unprecedented feats such as the atomic resolution electrostatic potential mapping of graphene sheets [9].

We utilize a different configuration of electron holography, inspired by a 1990 proposal by J. M. Cowley. As illustrated in Fig.1, the electron beam travels down the microscope column to the Condenser 2 aperture, where it diffracts through a highly efficient phase grating into electron probes with tens of nanometers spatial separation. The specimen is positioned such that all three diffraction probes initially pass through vacuum. The probes are then scanned such that the +1 order probe interacts with the specimen while the 0 and -1 order probes pass through vacuum. They are then recombined through the post specimen optics and interfere at the CCD camera.

We performed this experiment on an FEI 80-300 Titan TEM at $300 \mathrm{kV}$ in STEM mode. A $50 \mu \mathrm{m}$ diameter, $100 \mathrm{~nm}$ pitch grating is positioned in the $\mathrm{C} 2$ aperture plane. The test sample we used was graphitic carbon and gold nanoparticles on amorphous carbon. The interference fringes formed on the CCD camera created from such a setup should have the form $I_{i}\left(x_{0}\right) \equiv|\tilde{\psi}(k)|^{2}=\left|C_{0}\right|^{2}+\left|C_{-1}\right|^{2}+$ $\left|C_{+1} \cdot \chi\right|^{2}+2 C_{-1} C_{0} \cos \left(k x_{0}\right)+2 C_{+1} \cdot \chi\left(C_{-1} \cos \left(2 k x_{0}-\phi_{i}\right)+C_{0} \cos \left(k x_{0}-\phi_{i}\right)\right)$, where $C_{n}$ is the pre-specimen amplitude of the nth diffracted probe from the grating, i represents the image number (the location in the scan), $\chi$ is proportional to the ratio of electrons coherently transmitted through the specimen relative to the the total number of incident electrons and $\phi$ is the phase shift imparted by the sample. Notice that there are three sets of interference due to the use of three incident probes. The -1 and $0^{\text {th }}$ order probes interfere to form the first cosine term, the +1 and -1 order probes interfere to form the $2 \mathrm{kx}_{0}$ term and the +1 and $0^{\text {th }}$ order probes interfere to form the final term. Note that only the first of these three sets does not interfere with the specimen. We observed such fringes with the predicted varying frequency between $\mathrm{kx}_{0}$ and $2 \mathrm{kx}_{0}$ as shown in Figure $2(\mathrm{~b}-\mathrm{e})$.

To reconstruct the phase, we take the Fourier transform of the interference fringes and use the peak intensities to solve for $\phi$. We constructed a specimen with a smooth step function phase profile and used it to simulate a STEM-holography experiment. We then reconstructed the phase using a program we wrote that follows the above theory. Unlike other new phase contrast techniques, STEM-holographic contrast transfer function includes a non-zero DC component and therefore could be more easily 
interpretable. We've found that the reconstructed phase jump can be measured almost perfectly far from the step.

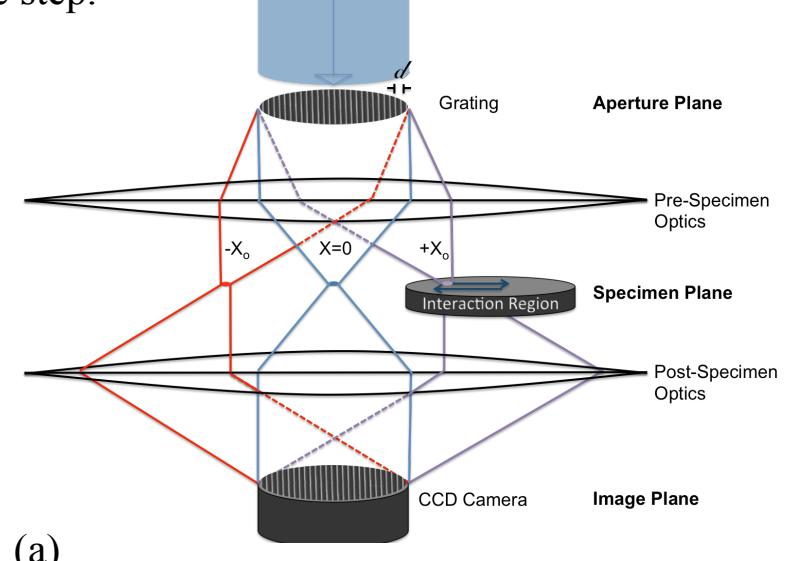

Figure 1. Experimental setup for STEMHolography. The electron beam is split into diffracted probes (three probes are shown, the $+1,0$ and -1 orders) by a diffraction hologram with pitch $\mathrm{d}=100[\mathrm{~nm}]$.
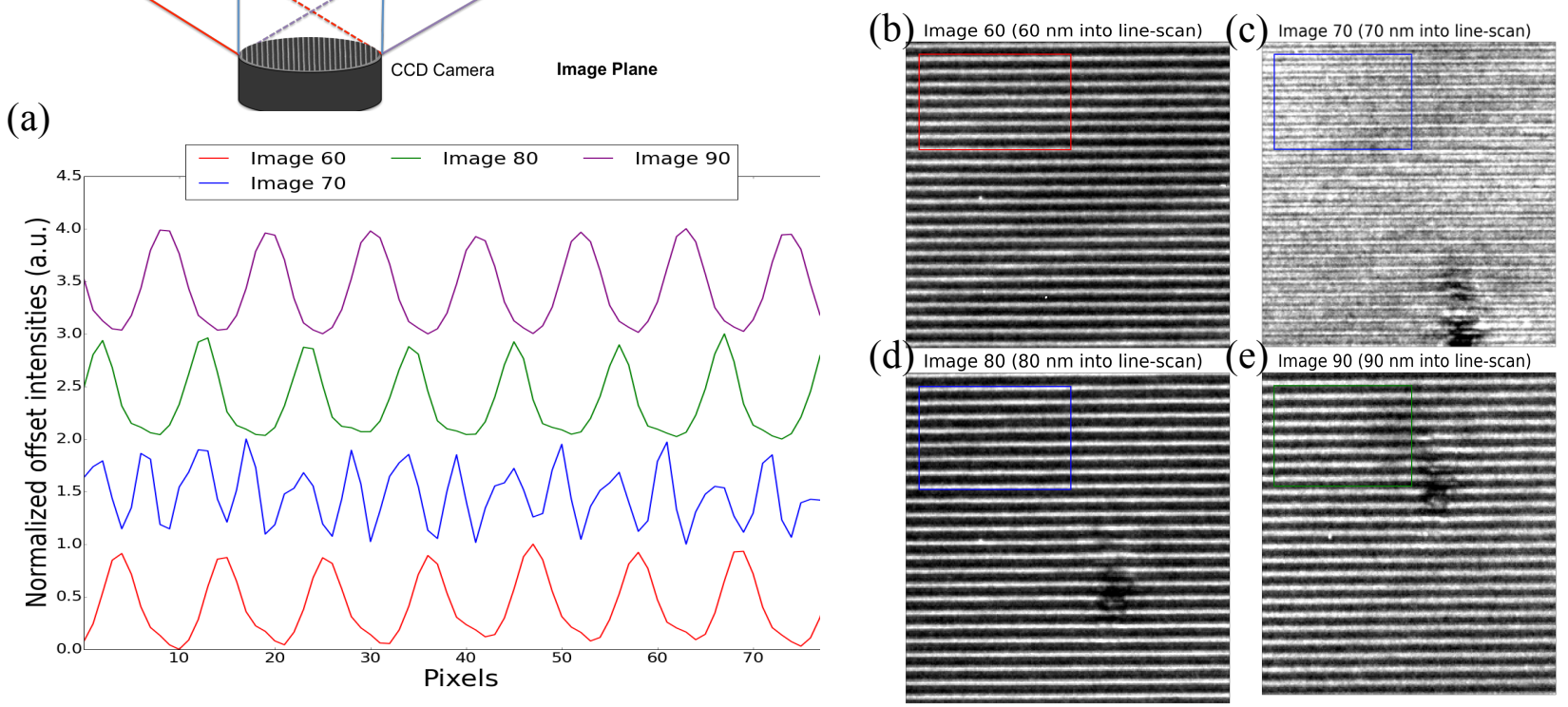

Figure 2. (a) Line profiles of images (b-e). (b-e) Interference fringes collected on the CCD camera while the +1 order probe is $60,70,80$ and $90 \mathrm{~nm}$ into a line scan, respectively. The boxed areas note the region from which the line profile was extracted. Note the agreement with the theory over different phase interactions, especially the frequency doubling in (c).

\section{References:}

[1] J. M. Cowley, Ultramicroscopy 34, 293 (1990).

[2] X.-c. Bai, et al., eLife 2, e00461 (2013).

[3] T. J. Pennycook et al., Ultramicroscopy 151, 160 (2015).

[4] M. Humphry, et al., Nat Commun 3, 730 (2012).

[5] H. Lichte, Advances in optical and electron microscopy 12, 25 (1991).

[6] A. Tonomura, Electron Holography (Springer-Verlag Berlin Heidelberg, 1993).

[7] M. R. McCartney et al., Annual Review of Materials Research 37, 729 (2007).

[8] P. A. Midgley, et al., Nature Materials 8, 271 (2009).

[9] D. Cooper, et al., Journal of Applied Physics 115, 233709 (2014).

[10] The authors acknowledge the use of CAMCOR facilities, as well as the U.S. Department of Energy, Office of Science, Basic Energy Sciences, under Award DE-SC0010466 as well as the National Science Foundation Graduate Research Fellowship Program under Grant No. 1309047. We also thank Josh Razink for instrument support. 